treatment. If the PRE is caused by some type of conditioned emotional response such as frustration (Amsel, 1958), it could be that ECS interferes in some way with the conditioned frustrative reaction, resulting in a loss of the PRE. This interference, however, appears to differentially affect VR-and FR-trained subjects. The combined findings suggest that reinforcement schedule is an important variable in determinining the effects of ECS, although it is not clear that a memory consolidation hypothesis would suggest it.

\section{REFERENCES}

Amsel, A. The role of frustrative nonreward in noncontinuous reward situations. Psychological Bulletin, 1958, 55, 102-119. Cooper, R. M., \& Koppenaal, R. J. Suppression and recovery of a one-triai avoidance response after a single ECS. Psychonomic Science, 1964, 1, 303-304.

Kohlenberg, R., \& Trabasso, T. Recovery of a conditioned emotional response after one or two electroconvulsive shocks. Journal of Comparative and Physiological Psychology, 1968, 65, 270-273.

Lewis, D. J. Sources of experimental amnesia. Psychological Review, 1969, 76, 461-472.

McGaugh, J L. Time-dependent processes in memory storage. Science, 1966, 153, 1351-1358.

Young, A. G., \& Galluscio, E. H. Effect of ECS on one-trial learning following continuous and partial reinforcement training. Psychonomic Science, 1970, 21, 43-44.

Young, A. G., \& Galluscio, E. H. Recovery from ECS-produced amnesia. Psychonomic Science, 1971, 22, 149-151.

(Received for publication November 13, 1974.)

\title{
Convergence and stereoscopic depth shifts produced by interocular delays in stimulation
}

\author{
EUGENE R. WIST \\ Whitely Psychology Laboratories, Franklin and Marshall College \\ Lancaster, Pennsylvania 17604
}

\begin{abstract}
The possibility that changes in perceived stereoscopic depth resulting from interocular delays in stimulation are due to small increases in the convergence of the eyes during the delay intervals was examined. An indirect method involving the measurement of fixation disparity was used to indicate possible convergence movements. It was found that the magnitude of the shift in perceived stereoscopic depth increased with increasing interocular delay interval as did the magnitude of convergence, but that the magnitude of the former was twice that of the latter. Therefore, the possibility that convergence shifts were responsible for depth shifts was ruled out. The alternative hypothesis that such depth shifts are the result of a central equivalency of binocular disparity and temporal disparity is consequently still tenable.
\end{abstract}

Previous studies (Wist, 1968; Wist, 1970; Wist \& Gogel, 1966) have shown that a temporal disparity in stimulation of the two eyes has an effect on perceived stereoscopic depth which is equivalent to that produced by an increase in binocular (spatial) disparity. This was demonstrated by presenting a luminous fixation stimulus which was binocularly viewed and continuously

This research was supported by Grant EY00268 from the National Eye Research Institute of the U.S. Public Health Service. This report was written while the author was a "Senior U.S. Scientist Awardee of the Alexander von Humboldt Foundation" at the Department of Neurophysiology, University of Freiburg, West Germany. illuminated and a luminous test stimulus which was dichoptically viewed and intermittently illuminated. The left eye was exposed to a 5-msec presentation of the test stimulus and after a variable interocular delay interval (IDI) of 0 to $96 \mathrm{msec}$, the right eye was exposed to the test stimulus for the same exposure duration. The binocular disparity between the test and fixation stimuli was set at 0 so that when IDI was 0 , the two stimuli appeared equidistant. It was found that if the IDI exceeded some $32 \mathrm{msec}$, the perceived depth interval between test and fixation stimuli changed, with the test stimulus appearing increasingly behind the fixation 
stimulus with increasing IDI.

One possible interpretation of this depth shift effect is that a genuine trading relation exists between temporal disparity (IDI) and spatial (binocular) disparity, such that the cortical areas involved in the analysis of stereoscopic depth, process the former as equivalent to the latter within certain temporal limits. However, in order for the existence of such a trading relation to be clearly established, it is necessary to demonstrate that the two eyes do not move with respect to each other during the IDI. If they do, then noncorresponding points would be stimulated by the test stimulus in each eye and the result would be an altered binocular disparity. If this occurred, the temporal disparity in stimulation would serve only to alter the proximal stimulus at the retinas, and no central, trading relation would need to be assumed. In order for eye movements to account for the depth shift effect, which is always in the rearward direction (Wist, 1968), it would be sufficient for the eyes to convergence slightly during the IDI. If, for example, the first 5 -msec presentation of the test object to the left eye initiated a rapid convergence movement of both eyes, the second presentation to the right eye, after a sufficiently long IDI, would stimulate a point on the retina located nasally with respect to the point which would have been stimulated in the absence of this movement. The result would be an increase in the binocular disparity between the test and fixation objects, placing the depth location of the former behind the latter, consistent with the experimental results. ${ }^{1}$

The purpose of this study was to test for the existence of such eye movements. If a change in the convergence of the eyes during the IDI is responsible for the depth shift effect, then it ought to be found that the functions relating depth shift magnitude and IDI on the one hand, and convergence shift magnitude and IDI on the other, are superimposed. If, however, depth shift magnitudes are greater than convergence shift magnitudes, then the view that there is a centrally mediated trading relation between temporal and binocular disparity is still tenable.

\section{METHOD}

\section{Subjects}

Seven male college students with normal phorias, visual acuities of at least 20/20 (corrected, if necessary), and stereoscopic acuities of at least $25 \mathrm{sec}$ of arc, all as measured with the Bausch and Lomb Orthorater, were paid for their services.

\section{Apparatus}

A fixation disparity task (Ogle et al., 1957) was employed to measure possible convergence movements of the eye during the IDI. The stimulus display used, which was located $4 \mathrm{~m}$ from the subject's eyes, is shown schematically in the insert of the figure. A binocularly viewed horizontal bar, $5^{\prime}$ wide and $206^{\prime}$ long, separated an upper luminous bar, $4^{\prime}$ wide and $30^{\prime}$ high viewed by the left eye only from a lower luminous bar, $4^{\prime}$ wide and $65^{\prime}$ high, viewed by the right eye only. The lateral position of the upper bar was adjustable by subject by means of a pair of selsyn motors. A dark spot at the left of the horizontal bar, 103' laterally from the fixed lower bar, served as a fixation point. The $20^{\prime}$ test disk was located $103^{\prime}$ to the left of the fixation point. A point of light, $30^{\prime}$ below the test disk, could be varied in depth under subject's control. The luminance of this point was adjusted so as to appear equal in perceived brightness to the test disk.

\section{Fixation Disparity Measurement}

The horizontal bar was continuously illuminated while the vertical bars and the test disk were intermittently illuminated for $5 \mathrm{msec}$ with a repetition interval of $1 \mathrm{sec}$. For the reference fixation disparity condition, the IDI for both the test disk and the vertical bars was set at 0 msec (measured from the offset of the first to the onset of the second flash). While fixating the point, the subject adjusted the lateral position of the upper bar until it appeared to be vertically aligned with the lower bar. The starting position of the upper bar was varied randomly, appearing to the left and right of the lower bar equally of ten. Fifty practice trials were given under these conditions before nonzero IDIs were introduced. On these latter trials, the test object and the upper vertical bar were presented to the left eye for $5 \mathrm{msec}$ followed by an IDI of either, $30,60,90$, or $120 \mathrm{msec}$ followed by presentation of the test disk and the lower vertical bar to the right eye for $5 \mathrm{msec}$. If the eyes converged during the IDI, then the upper bar would appear to have shifted to the right of the lower bar. Thus, in order to readjust the upper bar so as to appear aligned with the lower one again, the subject would have to move it to the left. The amount of displacement of the upper bar to the left required in order to compensate for its apparent shift to the right expressed in min of arc when IDI $>0$, minus the displacement required when IDI $=0$, constituted the measure of convergence. Divergent eye movements were indicated by compensatory adjustments of the upper bar to the right.

\section{Depth Shift Measurement}

These measurements were made under stimulus conditions identical to those for fixation disparity measurements. The subject's task for the reference condition was to adjust the distance of the light point below the test disk in depth so that it appeared equidistant to the test disk when IDI was set at zero. The starting position of the movable point was varied randomly appearing equally often both in front of and behind the tesk disk. Fifty practice trials were given under these conditions before nonzero IDIs were introduced. On these latter trials as for the fixation disparity measure, the test disk and the upper bar were presented simultaneously to the left eye, and then after an IDI of either $30,60,90$, or $120 \mathrm{msec}$, the test disk and lower bar were presented to the right eye. If the IDI were sufficiently long, the subject perceived a rearward shift in the depth location of the test disk in relation to the horizontal bar. His task was to readjust the position of the light point in depth so that it appeared to be as far behind the horizontal bar as the test disk. The difference in the mean depth location of this point when IDI $=0$ and when IDI $>0$, constituted the measure of the depth shift due to the temporal disparity in stimulation.

\section{Procedure}

A total of 121 -h sessions were required to collect the data. In the first session, subjects matched the perceived brightness of the vertical bars and the test disk for each eye to be equal to that of the $1.6-\mathrm{ml}$ horizontal bar. Two practice trials plus five additional trials were administered for each of these four stimuli in random order. The remainder of this session was used for 50 fixation disparity practice trials with $\mathrm{IDI}=0$. The second session was devoted to 50 depth shift practice trials with $I D I=0$. The third and fourth sessions each involved 20 practice trials on each task with IDI $>0(30,60,90$, and $120 \mathrm{msec})$. For sessions $8-12$, subjects received 10 IDI $=0$ and IDI $>0$ trials randomly 


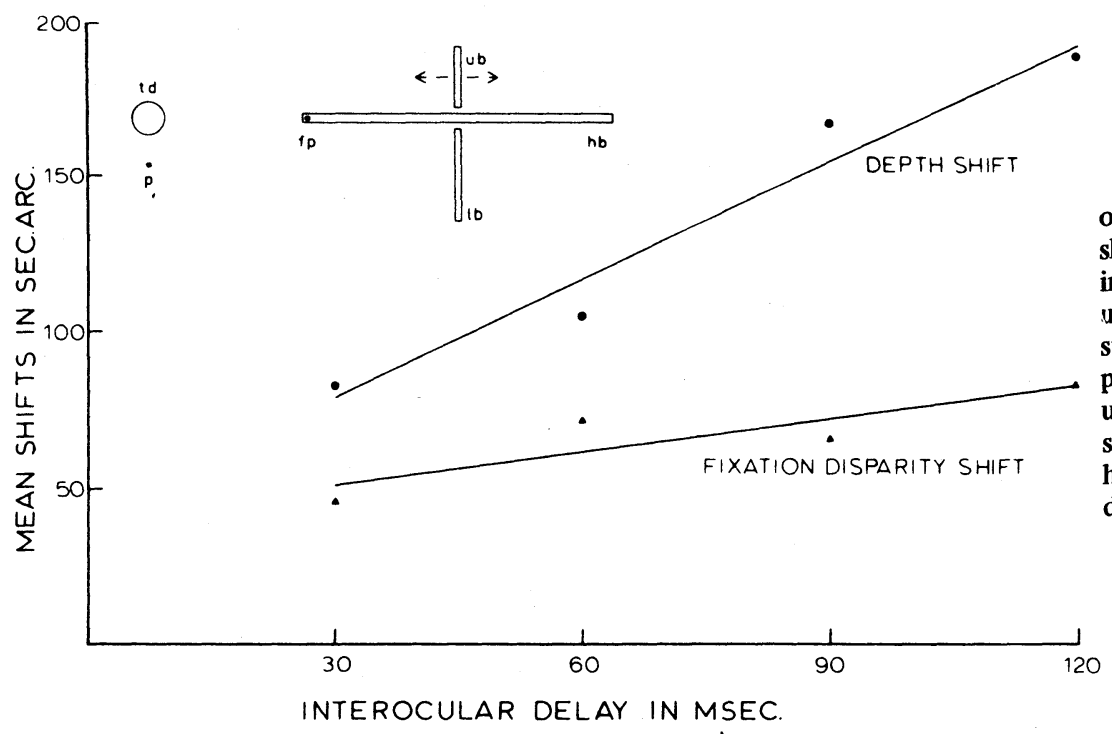

Figure 1. Mean shift in depth in sec of arc of the test disk and mean fixation disparity shift in sec of arc as a function of interocular delay interval in msec. Insert at upper left schematically represents the stimulus display. $t d=$ test disk, $p=$ light point, $\mathrm{fp}=$ location of fixation point, $\mathrm{ub}=$ upper vertical bar (laterally adjustable by subject), $l b=$ lower vertical bar, $h b=$ horizontal bar. See text for angular dimensions and separations.

distributed on each of the two tasks for a total of 320 trials $(40$ trials per session $\times 8$ sessions). Each subject was run during a different random order of stimuli presentation. The order of nonzero IDI was randomized in blocks of 10 trials each. Each session began with a 5-min period of adaptation to an adapting field whose luminance was $28 \mathrm{ml}$ and whose angular size was $80 \mathrm{deg}$ wide by $50 \mathrm{deg}$ high. During each session, two additional minutes of light adaptation were given after each five trials in order to insure that only the stimulus display was seen.

\section{RESULTS AND DISCUSSION}

The upper line in the figure represents the mean depth shifts for the seven subjects as a function of IDI. Each point represents the difference in sec.arc between the perceived depth interval between the test disk and the horizontal bar for the IDI given in the abscissa and for this depth interval when IDI was zero. Each point represents the mean of 140 difference scores $(20$ per subject $\times 7$ subjects). All depth shifts were in the rearward direction and increased with increasing IDIs, confirming earlier results (Wist, 1968; Wist, 1970; Wist \& Gogel, 1966). The dashed line represents the fixation disparity shift in sec.arc as a function of IDI. The points on this line were calculated in the same manner as those for the depth shift function. Each point represents, for a given IDI, the mean shift in fixation disparity from the reference condition where IDI was zero. All shifts were in the direction of greater convergence of the eyes.

An analysis of variance of these data revealed a significant effect of IDI on both depth and fixation disparity shift magnitudes $(p<.01)$ and significantly greater depth than fixation disparity shifts $(p<.005)$. Scheffé tests showed that while the three longest IDIs produced depth shifts significantly greater than that resulting from the 30 -msec IDI, only the $120 \mathrm{msec}$. IDI resulted in a significantly greater fixation disparity shift than the 30-msec IDI $(\mathrm{p}<.01)$.
Since depth shifts were significantly greater than fixation disparity shifts, the two functions in the figure cannot be regarded as superimposed. The mean depth shift was two times that of the mean fixation disparity shift on the average. Thus, the increase in convergence which occurred during the interocular delay interval was not large enough to account for the magnitude of depth shifts, and therefore the hypothesis that such movements are responsible for the depth shift effect is not supported. The alternative, and more interesting, possibility that a genuine central trading relation exists between temporal and binocular disparity in the determination of stereoscopic depth remains open.

\section{REFERENCES}

Ogle, K. N., Martens, T. G., \& Dyer, J. A. Oculomotor imbalance in binocular vision and fixation disparity. Philadelphia: Lea and Febiger, 1967.

Wist, E. R. The influence of the equidistance tendency on depth shifts resulting from an interocular delay in stimulation. Perception \& Psych ophysics, 1968, 3, 89-92.

Wist, E. R. Do depth shifts resulting from an interocular delay in stimulation result from a breakdown of binocular fusion? Perception \& Psychophysics, 1970, 8, 15-19.

Wist, E. R., \& Gogel, W. C. The effect of interocular delay and repetition interval on depth perception. Vision Research $1966,6,325-334$.

\section{NOTE}

1. While it is true that a congugate saccadic movement of the eyes to the left when the test stimulus is located to the left of the fixation stimulus would produce the same result as a convergent movement, the opposite result would be produced by such a movement if the test object was located to the right of the fixation stimulus. In this case, congugate saccadic eye movement toward the test stimulus during the IDI would result in stimulation by the test stimulus of a more temporal rather than a more nasal portion of the second eye and, therefore, a forward depth shift would be predicted. The occurrence of such movements as a possible mediator of the depth shift effect is ruled out by the observation that rearward shifts in the depth location of the test object occur regardless of whether it appears to the left or the right of the fixation object, and regardless of the order in which the two eyes are stimulated.

(Received for publicaton November 25, 1974:) 